\title{
Measurements of interrupter resistance: reference values for children 3-13 yrs of age
}

\author{
P.J.F.M. Merkus*, H.G.M. Arets", T. Joosten*, A. Siero", M. Brouha\# ${ }^{\#}$ J.Y. Mijnsbergen*, \\ J.C. de Jongste*, C.K. van der Ent ${ }^{\#}$
}

Measurements of interrupter resistance: reference values for children 3-13 yrs of age. P.J.F.M. Merkus, H.G.M. Arets, T. Joosten, A. Siero, M. Brouha, J. Y. Mijnsbergen, J.C. de Jongste, C.K. van der Ent. (C) ERS Journals Ltd 2002.

ABSTRACT: The interrupter technique is a convenient and sensitive technique for studying airway function in subjects who cannot actively participate in (forced) ventilatory function tests.

Reference values for preschool children exist but are lacking for children $>7 \mathrm{yrs}$. Reference values were obtained for expiratory interrupter resistance $(R$ int,e) in 208 healthy Dutch Caucasian children 3-13 yrs of age.

A curvilinear relationship between $R$ int,e and height was observed, similar to published airways resistance data measured by plethysmography. No significant differences in cross-sectional trend or level of $R$ int,e were observed according to sex. It was found that Z-scores could be used to express individual Rint,e values and to describe intra- and interindividual differences based on the reference equation: ${ }^{10} \log R$ int, $\mathrm{e}=0.645-0.00668 \times$ standing height $(\mathrm{cm}) \mathrm{kPa} \cdot \mathrm{L}^{-1} \cdot \mathrm{s}^{-1}$ and residual SD $\left(0.093 \mathrm{kPa} \cdot \mathrm{L}^{-1} \cdot \mathrm{s}^{-1}\right)$.

Expiratory interrupter resistance provides a tool for clinical and epidemiological assessment of airway function in a large age range.

Eur Respir J 2002; 20: 907-911.

\begin{abstract}
*Dept of Paediatrics, Division of Respiratory Medicine, Sophia Children's Hospital, Erasmus University Medical Centre Rotterdam, Rotterdam, and "Wilhelmina Children's Hospital, University Medical Centre Utrecht, Utrecht, the Netherlands.

Correspondence: P.J.F.M. Merkus, Dept of Paediatrics, Division of Respiratory Medicine, Sophia Children's Hospital, Erasmus University Medical Centre Rotterdam, PO Box 2060, 3000 CB Rotterdam, The Netherlands. Fax: 31104636772

E-mail: merkus@alkg.azr.nl
\end{abstract}

Keywords: Children, interrupter technique, lung function test, reference values

Received: August 82001

Accepted after revision: March 192002
The interrupter technique is one of the few lung function tests that can be used for assessment of airway calibre in young children [1-3]. With this technique, measurements of the resistance of the respiratory system (Rint) can be carried out quickly, with minimal cooperation of the child. Rint measurements have been shown to be reproducible [1, 4-6], sufficiently sensitive to detect (sub)clinical airway obstruction [6,7], and to correlate satisfactorily with measurements of airway resistance $[4,8,9]$. The technique can not only be used as a tool to screen for airway obstruction, but also to assess the responses to bronchodilating and bronchoconstricting agents $[1,3$, 10]. It is especially suitable for preschool children because it only requires passive cooperation. However, passive measurements of airway function may also be required for clinical research in older children or in older children who are unable to perform forced expiratory manoeuvres because of developmental disorders or neuromuscular disease. Reference data are available for young children $[6,7,11,12]$ and adults [13], but not for children of $>7 \mathrm{yrs}$ of age. Therefore, the aim of the present study was to expand the previous data set to better describe relationships between expiratory resistance and body size. Normal $R$ int values were obtained during expiration $(R$ int,e) in 208 healthy Caucasian children aged 3-13 yrs, from a general population. Measurements were preferably made during expiration rather than during inspiration because Rint,e appears to be more sensitive to detecting changes in resistance within children due to respiratory infections, and to discriminating better between children with and without respiratory symptoms or disease as compared to Rint values obtained during inspiration [6].

\section{Methods}

Data set

Rint,e measurements were carried out using identical equipment and the same measurement protocol in two sets of healthy Dutch Caucasian children aged 1-13 yrs, recruited from two daycare centres, two kindergartens and two elementary schools. Information on respiratory symptoms, eczema, allergy, parental smoking, doctor's diagnosis of asthma, and asthma medication was obtained using modified International Study of Asthma and Allergies in Childhood (ISAAC) questionnaires [14]. Children were included in the reference population when they had no respiratory symptoms in the month prior to, or during, the measurements. Exclusion criteria were: history of asthma, recurrent rhinitis, eczema, cardiorespiratory or other chronic disease, known anatomical abnormalities of 
the upper or lower airways, and vocal cord disorders. The authors wanted to obtain reference values from a normal population rather than an ideal population [15]. Therefore, mild respiratory symptoms not requiring medical care in the past and involuntary exposure to parental smoking without a history of respiratory symptoms or disease were not part of the exclusion criteria. The study and its protocol were approved by the medical ethics committees of the medical centres and by the principals and boards of the institutes involved. Informed consent was given by the parents of all participating children. When children refused to cooperate, no Rint,e measurements were attempted.

\section{Equipment}

Rint,e was assessed using the MicroRint (Micro Medical Ltd, Rochester, UK), as described previously [6]. Rint,e was calculated using the back extrapolation technique to $\mathrm{t}=0 \mathrm{~ms}$ after shutter closure during $100 \mathrm{~ms}$ [1]. Daily calibrations of pressure and flow (volume) were carried out using a manometer and a $2 \mathrm{~L}$ precision pump. All measurements were carried out with a filter (Micro Medical Ltd) in place to prevent contamination and dysfunction [16].

\section{Measurement protocol}

The protocol has been described previously [6]. After the supervisor of the children explained the purpose of the measurements, a measurement was demonstrated on the supervisor and subsequent measurements were carried out in groups of 2-4 children at a time, in a familiar and quiet room. Children were seated and no physical exercise was allowed during $10 \mathrm{~min}$ prior to the measurements. During measurements, children were instructed to breathe quietly, sitting upright while the cheeks and chin were supported from behind by the investigator. The head was positioned in slight extension and a nose clip was used. The position of the MicroRint was adjusted on a support arm to facilitate unobstructed breathing. A minimal number of five correct tracings (maximal 10) was obtained at the peak of expiratory tidal flow, because expiratory interruptions appear more sensitive in detecting airways obstruction than those during inspiration [6]. Tracings were rejected in the cases of tachypnoea, usage of the vocal cords, extreme neck flexion or extension, or leakage of the mouth piece. Tracings not showing the timing of interruption on the flow tracing or tracings with a horizontal or declining pressure signal suggesting leakage at the mouth or altered ventilation pattern, were also discarded [2] (fig. 1).

\section{Data analysis}

The individual $R$ int,e data were expressed as median values because individual data were not normally distributed [6]. Reference values for Rint,e were described based on a model assuming a linear or curvilinear relationship with the following standard independent variables: standing height, weight, and age. Because of physiological similarities between $R$ int and airway resistance obtained by plethysmography, the current authors hypothesised that an exponential model with standing height would create the best fit for the Rint,e data, as seen in published reference equations for airway resistance $(R \mathrm{aw})$ [17, 18]. Trends of residuals with
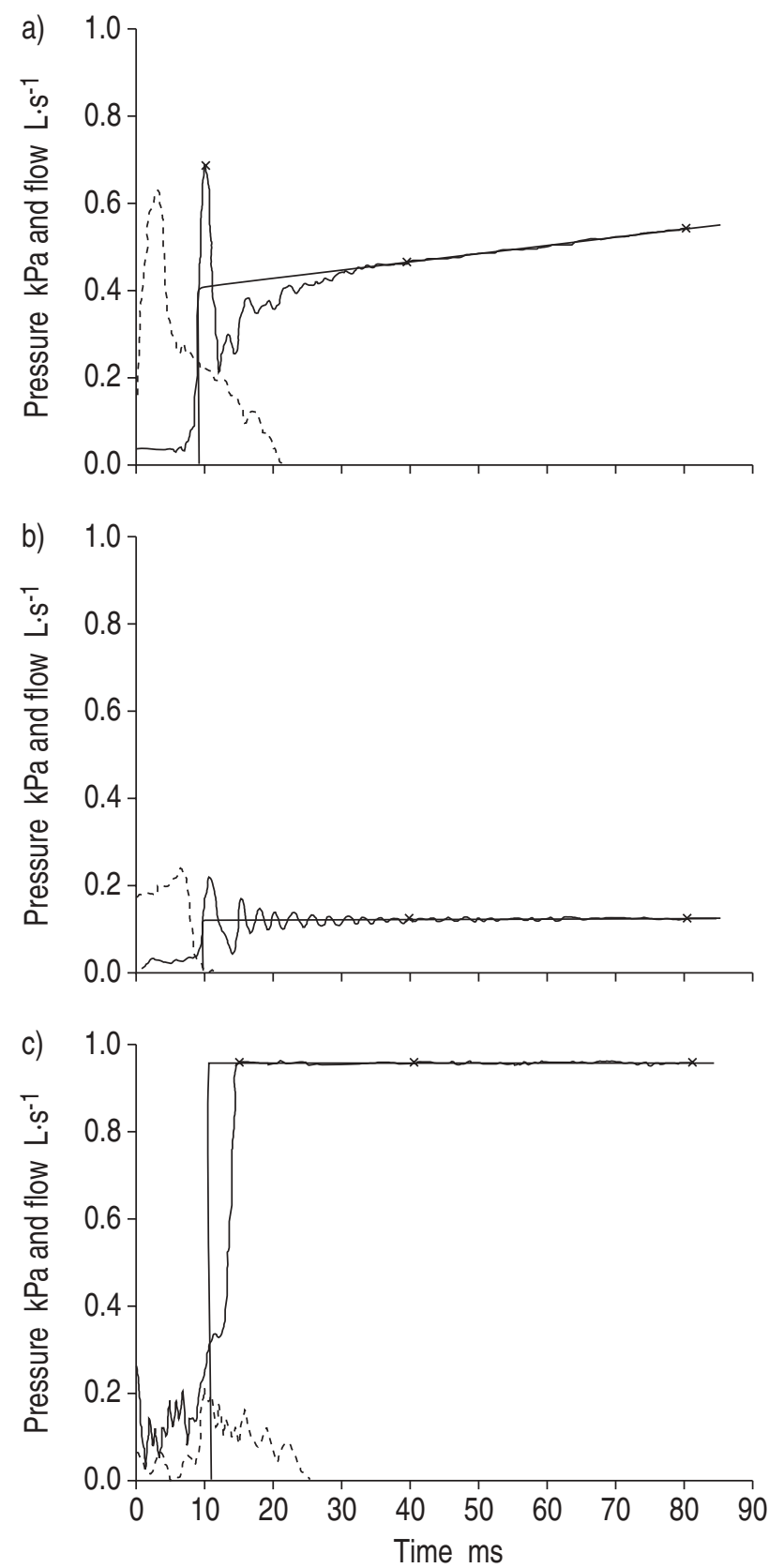

Fig. 1.-Examples of expiratory interrupter resistance (Rint,e) recordings. a) Correct manoeuvre, clearly visible timing of interruption on the flow tracing and approved interpolation and extrapolation of pressure signal. b) Visible leakage at the mouth, and a horizontal pressure signal following interruption. c) Flow signal affected by the usage of the vocal cords, and horizontal pressure. - - -: flow signal; - : pressure signal. 
height or age were assessed from linear regression analyses. The threshold for statistical significance was set at $\mathrm{p}=0.05$.

\section{Results}

\section{Subjects}

The first data set consisted of 135 healthy Dutch children (60 males) studied in Rotterdam, the Netherlands, who were selected from a survey in which the parents of 698 children were asked to participate. Permission was given for $341(49 \%)$ children, 36 refused participation, and 12 failed to complete the measurements. Of the remaining 293 children, 135 $(39 \%)$ met the inclusion criteria and completed the measurements. This included 54 healthy children described previously [6]. The second data set consisted of 79 Dutch children (41 males) studied in Utrecht, the Netherlands. These children were selected from a study in which parents of 445 children were asked to participate. Permission was obtained for 212 (48\%) of these children. Reliable Rint,e measurements were carried out in 200 children, of whom $79(40 \%)$ met the inclusion criteria listed above. Both studies were carried out in suburban parts of the cities, inhabited by middle class income Dutch families. Only two of the 24 children who failed to complete the measurements were $>4$ yrs of age. Anthropometric data of all

Table 1. - Anthropometric data of the reference population

\begin{tabular}{lcrr}
\hline Age range yrs (n) & Sex M:F & Height & Weight \\
\hline $1-3(6)$ & $4: 2$ & $88.2 \pm 6.8$ & $12.9 \pm 2.5$ \\
$3-4(8)$ & $3: 6$ & $104.9 \pm 6.2$ & $17.2 \pm 2.0$ \\
$4-5(17)$ & $7: 10$ & $109.5 \pm 3.7$ & $18.6 \pm 2.1$ \\
$5-6(28)$ & $12: 16$ & $116.4 \pm 4.3$ & $21.1 \pm 2.0$ \\
$6-7(29)$ & $12: 17$ & $119.8 \pm 4.9$ & $22.8 \pm 3.0$ \\
$7-8(25)$ & $13: 12$ & $129.9 \pm 4.6$ & $26.5 \pm 3.5$ \\
$8-9(29)$ & $8: 21$ & $133.1 \pm 7.5$ & $29.1 \pm 6.2$ \\
$9-10(21)$ & $15: 6$ & $142.3 \pm 5.0$ & $35.1 \pm 5.2$ \\
$10-11(17)$ & $10: 8$ & $149.1 \pm 4.3$ & $39.1 \pm 6.7$ \\
$11-12(24)$ & $11: 13$ & $154.7 \pm 6.7$ & $41.1 \pm 6.7$ \\
$12-13(8)$ & $6: 2$ & $156.7 \pm 10.6$ & $47.8 \pm 12.9$ \\
\hline
\end{tabular}

Data are expressed as mean \pm SD unless otherwise stated. M: male; F: female.

Table 2. - Differences between two data sets

\begin{tabular}{lcc}
\hline & $\begin{array}{c}\text { Data set 1, } \\
\text { Rotterdam }\end{array}$ & $\begin{array}{c}\text { Data set 2, } \\
\text { Utrecht }\end{array}$ \\
\hline Age yrs & $7.4 \pm 2.6$ & $8.4 \pm 2.9^{\#}$ \\
Standing height cm & $128 \pm 18$ & $133 \pm 20^{\#}$ \\
Weight kg & $27.2 \pm 9.3$ & $30.7 \pm 11.5^{\#}$ \\
M:F & $62: 76$ & $41: 38$ \\
Coefficient of variance & $11.7(1.2-21.4)$ & $6.9(0-35)$ \\
$\%$ median (range) & & \\
\hline
\end{tabular}

Data are presented as mean $\pm \mathrm{SD}$ unless otherwise stated. \#: $\mathrm{p}<0.03$, unpaired t-test.
214 children are summarised in table 1 . The children from data set 1 were slightly younger than those from data set 2 (table 2). The coefficients of variation from data set 1 were larger than those from data set 2 (table 2). This seemed to be explained by the differences in age between centres; at $<6$ yrs there was a negative correlation between coefficient of variation and age $(\mathrm{r}=-0.21, \mathrm{p}=0.004)$. The reference equation was based on the 208 children of $3-13$ yrs of age, as reliable measurements could only be obtained in six children $<3$ yrs.

\section{Reference equation for Rint}

An inverse, curvilinear relationship was found between $R$ int,e and the independent variables of standing height, age and weight. When standing height was used in an exponential model instead of a linear model with $R$ int,e, the explained variance increased from 59 to $63 \%$ and the residual standard deviation (RSD) decreased from 0.150 to $0.093 \mathrm{kPa} \cdot \mathrm{L} \cdot \mathrm{s}^{-1}$. Residuals of the exponential model were homoscedastically distributed, demonstrating no trend with standing height (fig. 2). When age was added to standing height in an exponential model, the explained variance increased by $<3 \%$, and the improvement of RSD was $<0.0003 \mathrm{kPa} \cdot \mathrm{L} \cdot \mathrm{s}^{-1}$. When age was used as the only independent variable in an exponential model with $R$ int,e, the explained variance was $64 \%$ (RSD= $\left.0.091 \mathrm{kPa} \cdot \mathrm{L} \cdot \mathrm{s}^{-1}\right)$, but the distribution of the residuals became heteroscedastic for subjects $>10 \mathrm{yrs}$ of age. In the children aged $>10$ yrs, the variability of standing height for age was larger than in the younger subjects. When using weight as the only independent variable, explained variance was $49 \%\left(\mathrm{RSD}=0.108 \mathrm{kPa} \cdot \mathrm{L} \cdot \mathrm{s}^{-1}\right)$.

Reference equations for Rint,e are:

$$
\begin{aligned}
R \text { int }, \mathrm{e}= & 1.927-0.00992 \times \text { standing height } \\
& (\mathrm{cm}) \mathrm{kPa} \cdot \mathrm{L} \cdot \mathrm{s}^{-1}
\end{aligned}
$$

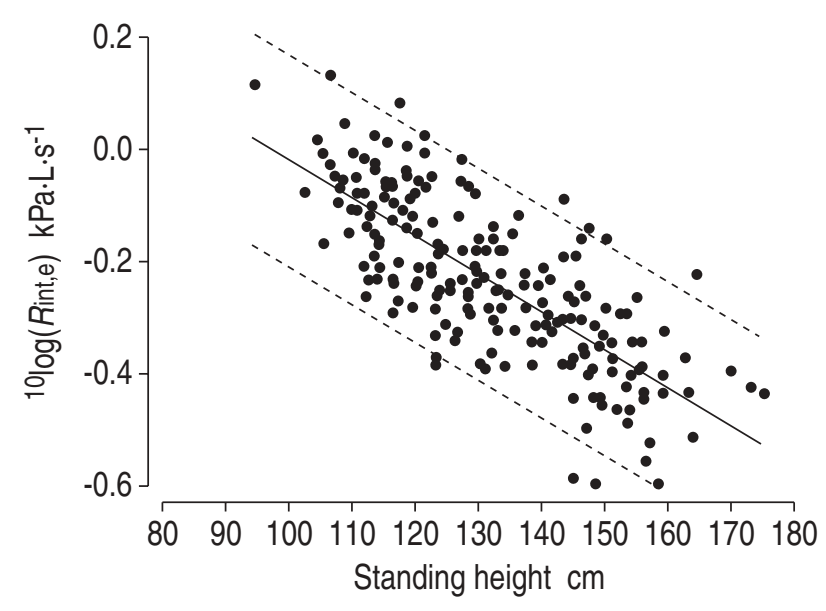

Fig. 2.-Relationship between ${ }^{10} \log ($ expiratory interrupter resistance (Rint,e)) and standing height for 208 children. - and ---: the exponential model (mean $\pm 95 \%$ confidence bands). 
for the linear model. $\mathrm{r}=-0.77, \mathrm{RSD}=0.150 \mathrm{kPa} \cdot \mathrm{L} \cdot \mathrm{s}^{-1}$ $(\mathrm{p}<0.001)$.

$$
\begin{aligned}
{ }^{10} \log \left(R_{\text {int }, \mathrm{e})=}\right. & 0.645-0.00668 \times \text { standing } \\
& \text { height }(\mathrm{cm}) \mathrm{kPa} \cdot \mathrm{L} \cdot \mathrm{s}^{-1}
\end{aligned}
$$

for the exponential model. $\mathrm{r}=-0.79, \mathrm{RSD}=0.093 \mathrm{kPa} \cdot \mathrm{L} \cdot \mathrm{s}^{-1}$ $(\mathrm{p}<0.001)$.

Based on the exponential model, the means (SEMs) of standardised residuals for 97 males and 111 females were $-0.079 \quad(0.096) \quad \mathrm{kPa} \cdot \mathrm{L} \cdot \mathrm{s}^{-1}$ and $0.071 \quad(0.097)$ $\mathrm{kPa} \cdot \mathrm{L} \cdot \mathrm{s}^{-1}$, respectively, with a mean $(95 \%$ confidence interval (CI)) difference between males and females of $0.15(-0.12-0.42) \mathrm{kPa} \cdot \mathrm{L} \cdot \mathrm{s}^{-1}$. No trends were observed between standing height and standardised residuals for males or females. The separate regression equations from the two data sets did not differ significantly in a multiple regression model that included centre $(\mathrm{p}=0.33)$ and interaction between standing height and centre $(\mathrm{p}=0.59)$.

\section{Discussion}

A few studies have reported reference equations for the interrupter technique in young school children [6, $7,11,12]$, but for older children these are lacking. Because of the possible applications of this technique in a larger age range (epidemiological and clinical research, children unable to participate in active lung function measurements), normal values for Rint,e were obtained in 208 healthy Caucasian children aged between 3-13 yrs. In a previous study by the current authors, a linear model was considered satisfactory to describe the relationship between height and Rint,e [6], but in the present study, due to the larger range in height, an exponential model appeared more appropriate because of a curvilinear relationship. This pattern is consistent with reports of Raw in healthy children [19].

This is the largest study of Rint,e that has been performed in healthy preschool and school children so far. Despite the large number of observations, a significant sex-related difference in airway patency was not detected. This suggests that a possible small difference in airways resistance between sexes is not clinically relevant, or that it cannot be detected when measuring resistance of the respiratory system with this technique.

Height, but not age, was used as an independent variable because of physiological arguments, not because the relationship between height and Rint,e was statistically superior. It is conceivable that body size can function as a proxy for airway calibre, whereas age may also indirectly reflect airway size in children $<13$ yrs but not in adolescents or adults. Age may be equally valid as a more convenient independent variable in reference equations for Rint,e [7], but this is probably limited to young children only. Indeed, in the present study, the variability of the residuals in the children $>10$ yrs of age was considerably increased compared to that in younger children, which is explained by a larger variation in height for age.
Because the residuals of the exponential model were normally distributed (with RSD $=0.093 \mathrm{kPa} \cdot \mathrm{L} \cdot \mathrm{s}^{-1}$ ), individual measurements can be expressed as Z-scores: $\frac{\mathrm{Z}=\left({ }^{10} \log (\text { measured } R \text { int,e })-{ }^{10} \log (\text { predicted } R \text { int,e })\right)}{\text { RSD }}(3)$

This facilitates comparisons within and between individuals. Until there is international standardisation for Rint,e measurements [20], reference equations are likely to differ according to the equipment and protocol of shutter timing and back extrapolation. In the protocol of the present study, interruptions were programmed at peak tidal expiratory flow, which appears to standardise inflation level [6,9]. The linear model of the present study fits remarkably well with the reference equation of VAN ALtena and Gimeno [13]:

$$
\begin{aligned}
R \text { int }= & 1.993-0.0092 \times \text { height }(\mathrm{cm})- \\
& 0.0009 \times \text { age }(\mathrm{yrs}) \mathrm{kPa} \cdot \mathrm{L} \cdot \mathrm{s}^{-1}
\end{aligned}
$$

van Altena and Gimeno [13] studied Rint in 172 adults and teenagers, although the exact measurement procedure was not described and the population and equipment differed markedly. The current results are not comparable with those of KLUG et al. [11] who programmed inspiratory interruptions at $50 \mathrm{~mL}$ above functional residual capacity. The effect of this procedure might have been that with increasing body size, interruptions occurred at progressively lower inflation levels. This could explain the lesser slope with height and lower explained variance of their reference equations.

It is difficult to compare Rint,e values with measurements of Raw or lung resistance $(R \mathrm{~L})$ in healthy populations because of differences in technique and population characteristics, but the reference equation present in this study compares favourably with those of Dab and Alexander [17] and Helliesen et al. [18], respectively:

$$
\begin{aligned}
& \log (\text { Raw })=0.712-0.0064 \times \text { height }(\mathrm{cm}) \\
& \log \left(R_{\mathrm{L}}\right)=0.627-0.0068 \times \text { height }(\mathrm{cm})
\end{aligned}
$$

The present authors were able to measure Rint,e reliably in only six children $<3$ yrs of age and do not recommend routine assessment of Rint,e in children of that age because of low feasibility [6]. The use of face masks in children $<3$ yrs may enhance the feasibility of the test, but Rint measurements obtained using face masks can differ from those obtained with mouthpieces [21] due to differences in the compliance and resistance of the mask and the degree of airways obstruction. Rint measurements using face masks may require specific reference equations.

The interrupter technique remains one of the most convenient and sensitive tests of airway function in young children $>3$ yrs of age, and has the potential for use over a wide age range.

Acknowledgements. The authors would like to thank all the children, parents, and personnel of the participating institutions for their enthusiastic contribution to this study. 


\section{References}

1. Phagoo SB, Wilson NM, Silverman M. Evaluation of a new interrupter device for measuring bronchial responsiveness and the response to bronchodilator in 3 year old children. Eur Respir J 1996; 9: 1374-1380.

2. Bridge PD, Rangantathan S, McKenzie SA. Measurement of airway resistance using the interrupter technique in preschool children in the ambulatory setting. Eur Respir J 1999; 13: 792-796.

3. Bisgaard H, Klug B. Lung function measurement in awake young children. Eur Respir J 1995; 8: $2067-$ 2075.

4. Phagoo SB, Watson RA, Pride NB, Silverman M. Accuracy and sensitivity of the interrupter technique for measuring the response to bronchial challenge in normal subjects. Eur Respir J 1993; 6: 996-1003.

5. Phagoo SB, Wilson NM, Silverman M. Evaluation of the interrupter technique for measuring change in airway resistance in 5-year old children. Pediatr Pulmonol 1995; 20: 387-395.

6. Merkus PJFM, Mijnsbergen JY, Hop WCJ, de Jongste JC. Interrupter resistance in pre-school children: measurement characteristics and reference values. Am J Respir Crit Care Med 2001; 163: 13501355.

7. McKenzie SA, Bridge PD, Healy MJR. Airway resistance and atopy in preschool children with wheeze and cough. Eur Respir J 2000; 15: 833-838.

8. Chowienczyk PJ, Lawson CP, Lane S, et al. A flow interruption device for measurement of airway resistance. Eur Respir J 1991; 4: 623-628.

9. Oswald-Mammosser M, Charloux A, Donato L, et al. Interrupter technique versus plethysmography for measurement of respiratory resistance in children with asthma or cystic fibrosis. Pediatr Pulmonol 2000; 29: 213-220.

10. Bridge PD, Lee H, Silverman M. A portable device based on the interrupter technique to measure bronchodilator response in schoolchildren. Eur Respir J 1996; 9: 1368-1373.

11. Klug B, Bisgaard H. Specific airway resistance, interrupter resistance, and respiratory impedance in healthy children aged 2-7 years. Pediatr Pulmonol 1998; 25: 322-331.

12. Lombardi E, Sly PD, Concutelli G, et al. Reference values of interrupter respiratory resistance in healthy preschool white children. Thorax 2001; 56: 691695.

13. van Altena R, Gimeno F. Respiratory resistance measured by flow-interruption in a normal population. Respiration 1994; 61: 249-254.

14. Asher MI, Keil U, Anderson HR, et al. International Study of Asthma and Allergies in Childhood (ISAAC): rationale and methods. Eur Respir J 1995; 8: 483-491.

15. Dezateux C, Wade A, Schmalisch G, Landau L. Maximizing effective research in infant respiratory function. In: Stocks J, Sly PD, Tepper RS, Morgan WJ, eds. Infant respiratory function testing. New York, Wiley Liss, 1996; pp. 521-550.

16. Frey U, Stocks J, Coates A, Sly P, Bates J. Standards for infant respiratory function testing: Specifications for equipment used for infant pulmonary function testing. ERS/ATS Task Force. Eur Respir J 2000; 16 : 731-740.

17. Dab I, Alexander F. Lung function measured with a whole body plethysmograph. Standard values for children and young acults. Acta Paediatr Belg 1979; 32: 259-267.

18. Helliesen PJ, Cook CD, Friedlander L, Agathon S. Studies of respiratory physiology in children. I. Mechanics of respiration and lung volumes in 85 normal children 5 to 17 years of age. Pediatrics 1958; 22: $80-93$.

19. Quanjer PhH, Stocks J, Polgar G, Wise M, Karlberg J, Borsboom G. Compilation of reference values for lung function measurements in children. Eur Respir J 1989; 2: Suppl. 4, 184s-262s.

20. Carter ER. It is time to consider standardizing the interrupter technique. Eur Respir J 1997; 10: 14281429.

21. Child F, Clayton S, Davies S, Fryer AA, Jones PW, Lenney W. How should airways resistance be measured in young children: mask or mouthpiece? Eur Respir J 2001; 17: 1244-1249. 